\title{
Wireless Underground Sensor Networks: Channel Modeling and Operation Analysis in the Terahertz Band
}

\author{
Mustafa Alper Akkaş and Radosveta Sokullu \\ Department of Electrical \& Electronic Engineering, Faculty of Engineering, Ege University, Bornova, 35100 Izmir, Turkey \\ Correspondence should be addressed to Mustafa Alper Akkaş; alper.akkas@ege.edu.tr
}

Received 17 October 2014; Revised 27 January 2015; Accepted 10 February 2015

Academic Editor: Mugen Peng

Copyright ( 2015 M. A. Akkaş and R. Sokullu. This is an open access article distributed under the Creative Commons Attribution License, which permits unrestricted use, distribution, and reproduction in any medium, provided the original work is properly cited.

\begin{abstract}
Wireless underground sensor networks (WUSNs) are networks of sensor nodes operating below the ground surface, which are envisioned to provide real-time monitoring capabilities in the complex underground environments consisting of soil, water, oil, and other components. In this paper, we investigate the possibilities and limitations of using WUSNs for increasing the efficiency of oil recovery processes. To realize this, millimeter scale sensor nodes with antennas at the same scale should be deployed in the confined oil reservoir fractures. This necessitates the sensor nodes to be operating in the terahertz $(\mathrm{THz})$ range and the main challenge is establishing reliable underground communication despite the hostile environment which does not allow the direct use of most existing wireless solutions. The major problems are extremely high path loss, small communication range, and high dynamics of the electromagnetic (EM) waves when penetrating through soil, sand, and water and through the very specific crude oil medium. The objective of the paper is to address these issues in order to propose a novel communication channel model considering the propagation properties of terahertz EM waves in the complex underground environment of the oil reservoirs and to investigate the feasible transmission distances between nodes for different water-crude-oil-soil- $\mathrm{CO}_{2}$ compositions.
\end{abstract}

\section{Introduction}

As defined in [1-3] WUSNs are networks of wireless sensor nodes operating below the ground surface. Compared to the current underground sensor networks which use wired communication methods for network deployment, WUSNs have advantages in timeliness of data, ease of deployment and data collection, concealment, reliability, and coverage density [1-3]. As a natural extension to the well-established wireless sensor networks (WSNs) [3] paradigm, WUSNs can be deployed to operate in underground tunnels and mines. In the future they are also envisioned to provide real-time monitoring capabilities in new challenging underground environments such as soil medium and oil reservoirs. In the tunnels and mines the WSNs are not fully buried undergroundthey operate below the surface of the earth but communicate through air ducts as medium. In the latter case, networks are fully located underground and communication takes place through the mixture of gas, water, and crude oil inside the oil reservoirs. These specific WUSNs are a promising and continuously expanding field that will enable a wide variety of novel applications, not possible with current underground monitoring techniques.

The system architecture of general WUSNs in soil medium is detailed in $[1,2]$. In this paper, we focus on WUSNs specifically tailored for operation in oil reservoirs. The envisaged system architecture of WUSNs in oil reservoirs is illustrated in Figure 1. The wireless underground sensor network for oil recovery consists of a base station (data sink) located at the wellbore and a large number of wireless sensor nodes deployed uniformly in the fractures of the oil reservoir. The wireless sensor nodes are injected into the fracture with the fluid during the hydraulic fracturing process and remain in the fractures fixed to their walls. These fractures are usually quite long $(\sim 100 \mathrm{~m})$ and very narrow $(1 \sim 3 \mathrm{~cm})$. The method proposed in this work for realizing the communication between the sensor nodes in these fractures is based on electromagnetic waves (EM) propagation which is completely different from other methods proposed so far [4-8]. The transmission environment in this case consists of 


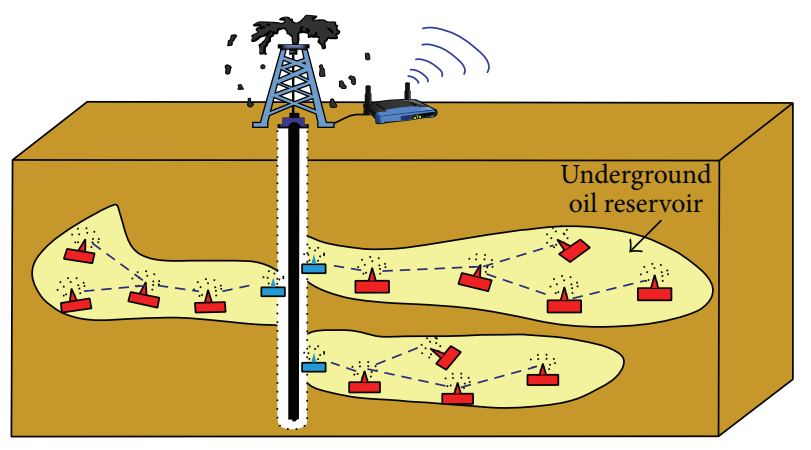

Underground wireless sensor

Underground sink sensor

Aboveground gateway

FIGURE 1: System architecture of the wireless sensor network for oil recovery.

a complex mixture of soil, water, and crude oil in various proportions and is very different from the traditional wireless propagation channel. As a result, the existing techniques for wireless propagation and communication fail to operate in a satisfactory manner in the fractures of the oil reservoir.

The major specifics of the WUSN operating in oil reservoirs can be summarized as follows.

(i) The environment where the wireless sensor nodes are deployed is a $3 \mathrm{D}$ hydraulic fracture with a size of $100 \mathrm{~m} * 3 \mathrm{~m} * 1 \mathrm{~cm}$ (length $*$ height $*$ width). As the width of the fracture is less than $1 \mathrm{~cm}$, the size of the sensor nodes should be in the millimeter magnitude. Furthermore, the millimeter scale sensor nodes necessitate antennas at the same scale which implies that the operating frequency has to be in the Gigahertz or Terahertz range [1-3].

(ii) The fractures are filled with thick fluid consisting of water and crude oil. In such fluid transmission medium, an EM signal with frequency in the Gigahertz and Terahertz range will experience severe medium absorption, which leads to extremely high path loss and short transmission radius.

(iii) The wireless sensor network as a whole will encounter severe energy problems. On one hand, the millimeter scale sensors only allow a very miniature battery with extremely limited energy supply. On the other hand the well-known solar and wind based energy harvesting schemes are not applicable underground.

The above-mentioned specifics of WUSN and the challenging problems they pose necessitate the development of new low-power signal propagation models and techniques for these networks.

In our previous work [1] we have studied in detail the propagation characteristics of electromagnetic (EM) waves in the Terahertz $(\mathrm{THz})$ band $(0.1-120.0 \mathrm{THz})$ through crudeoil/water mixture and soil. In this paper, we have extended our work to examine EM-based WUSNs communication techniques for enabling efficient communication in complex underground environments. Based on the determination of a transmission window in the Terahertz band optimal for the complex underground environment found in oil reservoirs the main contributions of this paper are as follows: proposition of a novel communication channel model considering the propagation properties of EM waves in the complex underground environment of the oil reservoirs; estimation of the feasible transmission distances between nodes and performance evaluation in terms of BER for different watercrude-oil-soil- $\mathrm{CO}_{2}$ compositions.

From here on the paper is organized as follows. In Section 2 we discuss studies related to oil recovery methods and elaborate on the specifics and limitations of using the $\mathrm{THz}$ band EM waves for underground communication. Section 3 analyzes the absorption properties of water, crude oil, carbon dioxide, and soil. Section 4 presents the suggested channel model for wireless communications in complex environments, consisting of mixtures of crude oil, water, $\mathrm{CO}_{2}$, and soil. Section 5 discusses the channel capacity and provides a performance evaluation based on the suggested channel model. Finally, the paper is concluded in Section 6.

\section{Related Work}

2.1. Extracting Information in Oil Recovery. Oil recovery is a very complex process and since a lot of effort and resources are involved it is very important to have as much information as possible about the oil recovery reservoir/field at each stage of the process. Thus researchers have been looking for different innovative ways to address the question of extracting information about the state of the resources from the hostile underground oil environment.

Liu et al. [4] study the progress of carbon dioxide in oil reservoirs using ultrasound and show that their approach may lead to unique and powerful ways of monitoring the position of carbon dioxide, as well as visualizing the reality of the oil producing area. The work reports results from different experiments related to the investigation of acoustic waves properties in different media. Their studies include ultrasound reflection properties of stationary waterair, water-oil, air-oil, and oil-oil interfaces, as well as those of moving interfaces. Furthermore, experiments of ultrasound reflection properties in porous medium combined with air, water, and oil are described.

In [5] Xu et al. describe an oilfield associated gas recovery device designed to take into consideration the status of associated gas recovery process. The relation between gas temperature and pressure in the device during the heating process is analyzed and calculated through the ideal-gas equation. The gas recovery device described is theoretically proved to be feasible. The oilfield associated gas can be effectively recovered by the device which requires low investment and convenient field application.

In their project Mazzini et al. [6] study the feasibility of using a robotic manipulator for tactile mapping. A method is developed that requires only robot joint encoders and avoids 
the use of any force or tactile sensors, which are complex and unreliable in such a hostile environment.

2.2. Using WUSNs in Oil Recovery Processes. Compared to the above-mentioned methods and techniques the use of WUSN for oil reservoir exploration is a very new and promising trend. However, due to the extremely high path loss, small communication range, and high dynamics of EM waves when penetrating the soil, water, and crude oil medium, most existing wireless solutions are inapplicable.

The oil recovery filed presents an underground environment which is much different from the ones studied in the works mentioned above. It contains besides soil, with different degrees of moisture, large amounts of crude oil as well as carbon dioxide (additionally injected in the fractures during the oil recovery process). The different medium components impose different attenuation and phase shifting effects on the EM waves especially when the Terahertz band is considered. The path loss fluctuates as the operating frequency changes due to the summation of EM waves with different phases. In order to achieve wireless transmission it is desirable to determine the frequency bands where the absorption loss experiences deep fades so that the path loss is minimized and the transmission range is maximized. As we are dealing with extremely high frequencies, even a very narrow channel (portion of the spectrum with deep fading in the values of the absorption) can bring more than enough bandwidth. However, since the EM waves are transmitted inside the fracture, the walls of the fracture limit the spread of the signal energy by reflecting the signal back into the fracture medium, which may also add additional fluctuations in the path loss values [1]. Furthermore, some molecules like carbon dioxide and hydrocarbons present in the considered medium are excited by EM waves at specific frequencies within the Terahertz band. An excited molecule internally vibrates; that is, its atoms show periodic motion while the molecule as a whole has constant translational and rotational motions. As a result of this vibration, part of the energy of the propagating wave is converted into kinetic energy or, from the communication perspective, is simply lost. EM waves at these specific frequencies within the Terahertz band encounter much higher attenuation in soil and liquid compared to air. This severely hampers the communication quality. Therefore, advanced models are necessary to accurately and completely characterize the underground channel in the Terahertz band and to lay out the foundations for efficient underground communication networks.

2.3. Channel Modelling for Underground Environments. One of the major issues for realizing WUSNs is evaluating the performance of the wireless channel existing between the nodes. A number of factors-frequency, propagation loss, scattering, refraction, reflection, diffraction, and so forthaffect the wireless transmission making it difficult to predict the received signal. Wireless channel modeling in general is a challenging task and has been addressed by researchers from very different aspects depending on the specific application in mind-from cellular systems to connecting medical imaging devices in hospitals $[9,10]$ and underground sensor networks. Channel modelling and simulation are an extremely important tool for analysis that reduces the cost of developing a complex system by limiting the amount of hardware required for evaluating its performance. These theoretical models have a second advantage in their ability to reproduce a channel for comparison between various communication strategies, resulting in an accurate measure of relative network performance as a whole. WUSNs are a very new concept and there are only very few studies that address the issues of the underground communication channel $[2,3,6]$. The first works on studying the properties of the wireless underground channel $[2,3]$ focus on the characterization of the "underground-underground," "undergroundaboveground," and "aboveground-underground" electromagnetic waves channels, while capturing the effects of environment parameters such as soil composition and soil moisture, and system parameters such as the antenna gain, operating frequency, and the sensor burial depth. The propagation characteristics are investigated through simulation and results are provided for the path loss between two adjacent nodes.

A later work that expands the model specifically for soil propagation is presented in [7]. The author suggests the "Soil Subsurface Wireless Communication (SSWC) channel model" based on extensive empirical study in a large agriculture field. The results of the experiments provide important insights into the model, which have not been available before. The SSWC channel model captures the signal attenuation and bit error rate (BER) in underground soil settings.

Another recently published work [8] explores the possibility of utilizing the method of magnetic induction to provide wireless communication in underground oil reservoirs. The work addresses the system architecture and operational framework of a magnetic induction- (MI-) based wireless sensor network, where both the wireless energy transfer and the wireless communications in the reservoir are realized by the magnetic induction technique. It analytically examines the feasibility of this method and proves that it can provide communication up to around $1.5 \mathrm{~m}$. Even though theoretically this is a promising approach, a feasible implementation seems possible in the next decades.

Working with underground sensors and using WUSNs in the $\mathrm{THz}$ band to provide detailed information during different phases of the oil recovery process are a very revolutionary topic and is not covered in any of the existing studies.

The objective of our work is to address the unique and important challenges for the implementation of wireless sensor networks in underground environments during oil recovery processes. To the best knowledge of the authors at the time of preparing this material there is no completed study regarding the modeling and thorough theoretical examination of the wireless communication process in WUSNs used in oil recovery process. We investigate the channel characteristics of the Terahertz EM waves in oil reservoir environments to determine an optimal frequency band that minimizes the total path loss and maximizes the 
transmission radius for an underground sensor network. Once the optimal operating frequency is determined, the link capacity and performance are evaluated based on the propagation channel model described in this work.

\section{Absorption Losses for Terahertz EM Waves in Water, Crude Oil, Carbon Dioxide, and Soil}

In this section the absorption properties of the different components comprising the underground oil reservoir environment, namely, water, salty water, crude oil, carbon dioxide, and soil, are examined in detail and the respective absorption loss graphs as a function of the frequency are derived. As a result, for each component, the suitable transmission window for wireless sensor nodes operating in the Terahertz band is determined.

3.1. Absorption Loss in Pure Water. Absorption spectrum of water at various temperatures in the whole infrared (IR) region $\left(0<\right.$ wavenumber $\left.\widetilde{v}<4000 \mathrm{~cm}^{-1}\right)$ can be obtained from ATR (attenuated total reflection) spectra recorded in the MIR (mid-infrared) region combined with absorption spectra measured in the FIR (far-infrared) region. Then, the absorption spectrum $L_{\mathrm{abs}}^{\text {water }}$ water (in $\mathrm{dB}$ ) of the EM waves in a sample of pure water within distance $d$ can be calculated according to the Beer-Lambert law $[1,11,12]$ :

$$
L_{\mathrm{abs}}^{\mathrm{water}}=k_{\mathrm{water}}(f) \cdot d \cdot 10 \log _{10}(e) \text {, }
$$

where $d$ is the transmission distance; $k_{\text {water }}(f)$ is the absorption coefficient, which is a function of the operating frequency and is influenced by the liquid temperature. The value of $k_{\text {water }}(f)$ and temperature can be looked up in [11], and $e$ is the well-known constant accepted as the base of the natural logarithm, equal to 2.71828 .

Based on (1), we can numerically analyze the properties of the water medium as a wireless channel for the Terahertz EM waves. Our results, given in Figure 2, show that there exist several transmission windows from 30 to $45 \mathrm{THz}$ and from 65 to $85 \mathrm{THz}$ where the absorption loss is acceptable for the realizing communication.

Further on, the transmission window 70-85 THz is examined in more detail (see Figure 3). For different temperatures (from $-4^{\circ} \mathrm{C}$ to $80^{\circ} \mathrm{C}$ ) it demonstrates absorption loss of less than $100 \mathrm{~dB}$. Especially for $78 \mathrm{THz}$ and temperatures above $25^{\circ} \mathrm{C}$ the absorption loss is less than $40 \mathrm{~dB}$.

Above $25^{\circ} \mathrm{C}$ the absorption loss for frequencies from 75 to $80 \mathrm{THz}$ is below $20 \mathrm{~dB}$. Thus the bandwidth of the feasible communication range for the Terahertz EM wave in water medium can be defined, depending on the system parameters, from 5 to $15 \mathrm{THz}$.

3.2. Absorption Loss in Salty Water. The discussion so far was related to the absorption spectrum of pure water; however

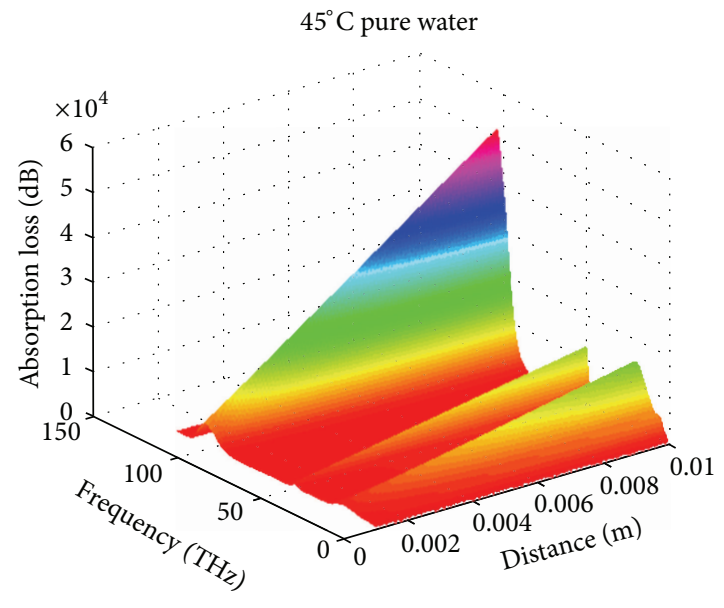

Figure 2: Absorption loss of pure water at $1 \mathrm{~mm}$ to $10 \mathrm{~mm}$ distance between 0.1 and $120 \mathrm{THz}$.

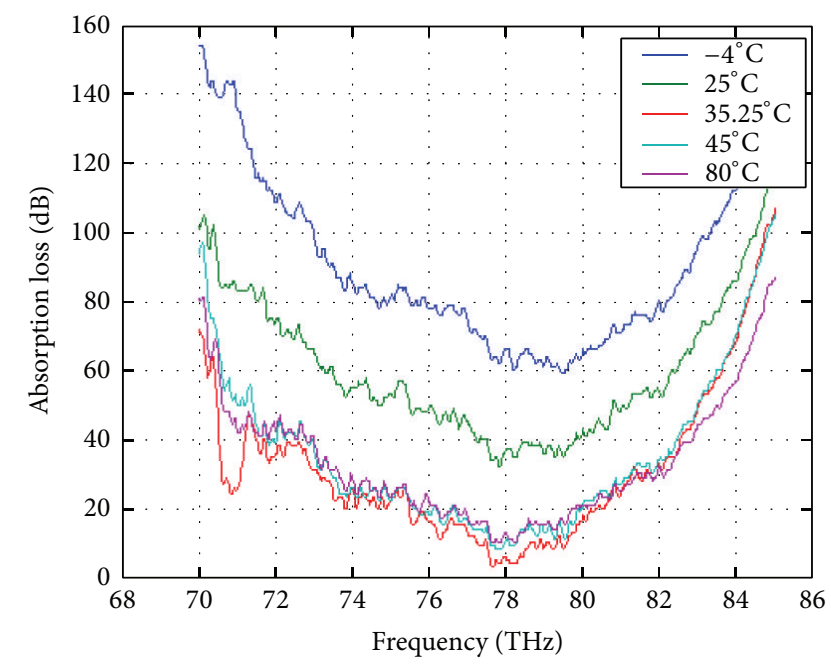

Figure 3: Absorption loss of pure water at $1 \mathrm{~mm}$ distance between 70 and $85 \mathrm{THz}$.

in oil recovery processes often sea water is also used. The absorption spectrum of salty water can be analyzed using

$$
\begin{gathered}
\alpha^{\prime}=\frac{4 \pi \kappa}{\lambda_{0}}, \\
\alpha^{\prime}=\alpha \cdot \log _{e}(10), \\
\alpha=\frac{4 \pi \kappa}{\lambda_{0} \cdot \log _{e}(10)}, \\
T=\frac{I}{I_{0}}=10^{-\alpha \ell} .
\end{gathered}
$$

The absorption coefficient, $\alpha^{\prime}$, can be expressed in terms of the imaginary part of the refractive index, $\kappa$, and the wavelength of the light (in free space), $\lambda_{0} . I_{0}$ and $I$ are the intensity (or power) of the incident light and the transmitted light, respectively, and $\ell$ is the distance the light travels through the material. There are two commensurate definitions of the 


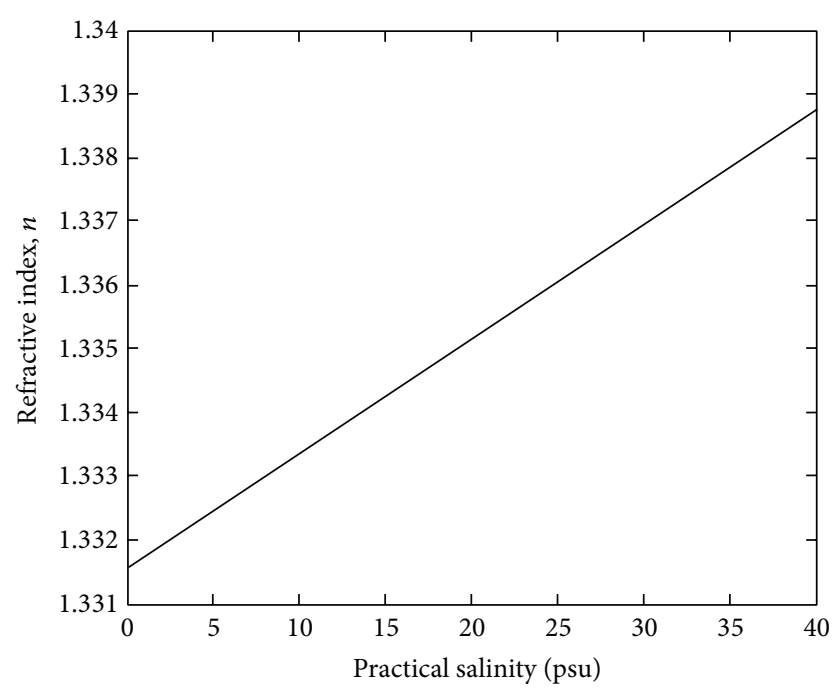

FIgURE 4: Refraction index values for different salinity [13].

absorption coefficient: for the case with gases the value of $\left(\alpha^{\prime}\right)$ is $\ln 10$ times (approximately 2.3 times) as large as the corresponding value for liquids $(\alpha)$ which is the one shown in (3). When the salinity of the water changes this leads to a change in the value of the refractive index $\kappa$ as shown in Figure 4 [13]. So, considering the Beer-Lambert law for liquids and combining (4) and (5), the absorbance of the electromagnetic waves as a function of both the salinity and the frequency is determined based on the following:

$$
A=\frac{1}{T\left(\lambda_{0}, \kappa, \ell\right)} .
$$

Using (6) and the values of the refraction index of sea water as provided in [13] for $22^{\circ} \mathrm{C}$ the absorption loss for pure and salty water is compared for frequencies from 70 to $85 \mathrm{THz}$ and plotted in Figure 5, for a sample with length $\ell=1 \mathrm{~cm}$.

The results show that there is a minimal difference less than $1 \%$ between the absorption in pure water (refraction index 1.332$)$ and that in salty water (40\% salinity where the refraction index is 1.339) for any given frequency in that range. So, analogous to the case with pure water (Section 3.1), minimum absorption loss in salty water is observed in the frequency range from 77 to $80 \mathrm{THz}$.

It should also be pointed out that similar results regarding the comparison between absorption loss in pure and salty water but for a different frequency spectrum have been obtained by the authors of [14].

3.3. Absorption Loss in Crude Oil. Following the same pattern as in the analysis for the water medium, in this section we analyze the absorption loss caused by the crude oil. It can generally be defined as

$$
L_{\mathrm{abs}}^{\mathrm{oil}}=k_{\mathrm{oil}}(f) \cdot d \cdot 10 \log _{10}(e),
$$

where $k_{\text {oil }}(f)$ is the absorption spectrum of crude oil and $d$ is the distance. Defining the absorption spectrum of oil $k_{\text {oil }}(f)$ is the key element and it is explained in more detail below.

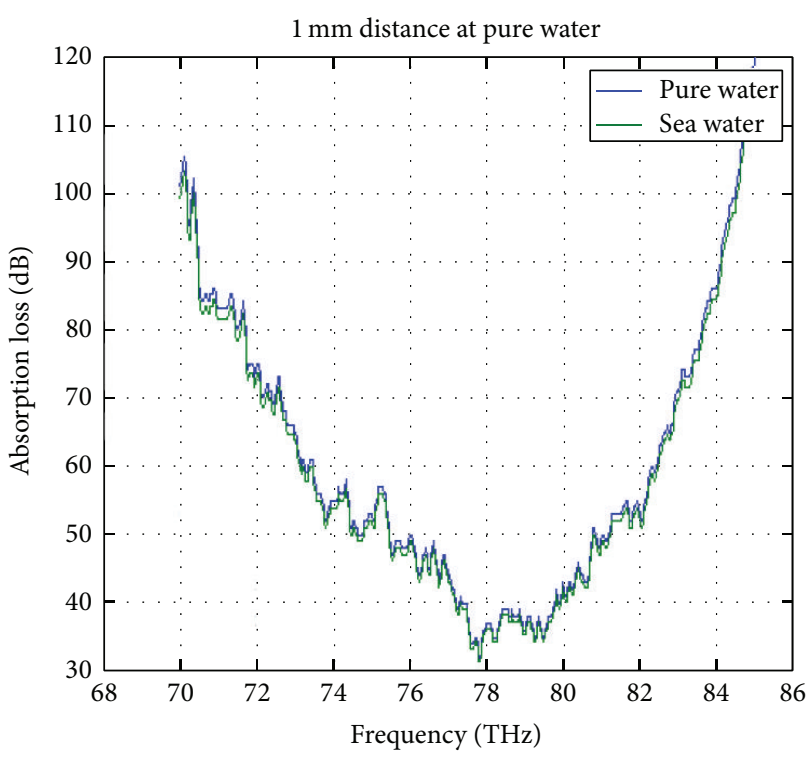

FIGURE 5: Absorption differences between pure water and $41 \%$ salinity sea water at $25^{\circ} \mathrm{C}$.

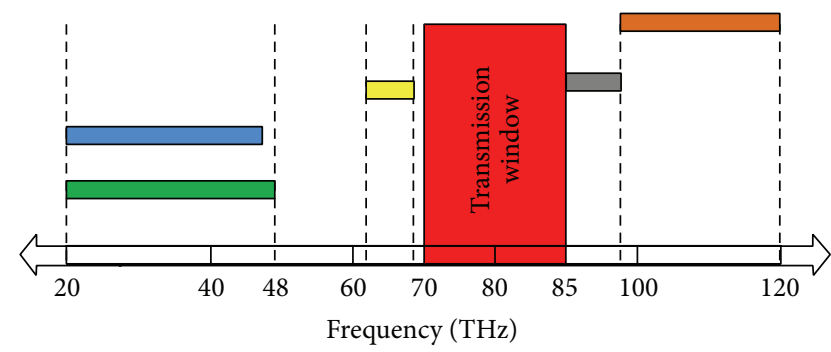

\footnotetext{
$\square$ Bending vibrations $(\mathrm{C}=\mathrm{C}, \mathrm{C}=\mathrm{O}, \mathrm{C}=\mathrm{N}$ )

$\square$ Stretching vibrations $(\mathrm{C}-\mathrm{C}, \mathrm{C}-\mathrm{O}, \mathrm{C}-\mathrm{N})$

$\square$ Stretching vibrations $(\mathrm{C}=\mathrm{N}, \mathrm{C} \equiv \mathrm{C})$

$\square$ Stretching vibrations $(\mathrm{C}-\mathrm{H})$

Stretching vibrations $(\mathrm{O}-\mathrm{H}, \mathrm{N}-\mathrm{H})$

Transmission window
}

FIGURE 6: IR spectroscopy correlation table with vibration and transmission windows.

Studying the absorption properties of crude oil requires the utilization of infrared (IR) spectroscopy. Crude oil is a mixture of a large number of different hydrocarbons each with different molecular structure. As mentioned in Section 2 excited molecules and pair of molecules internally vibrate in specific patterns causing part of the energy of the propagating wave to be converted into kinetic energy and thus the EM waves at these specific frequencies within the Terahertz band encounter much higher attenuation than at others. Figure 6 presents the summary of the IR spectroscopy results related to the type of vibration that causes deep absorptions and the respective frequencies. It clearly shows the two windows with min vibrational activities $(48-60 \mathrm{THz}$ and $70-85 \mathrm{THz})$.

Based on our previous work [15], we can state that the derived conclusion can be generalized for most types of crude 


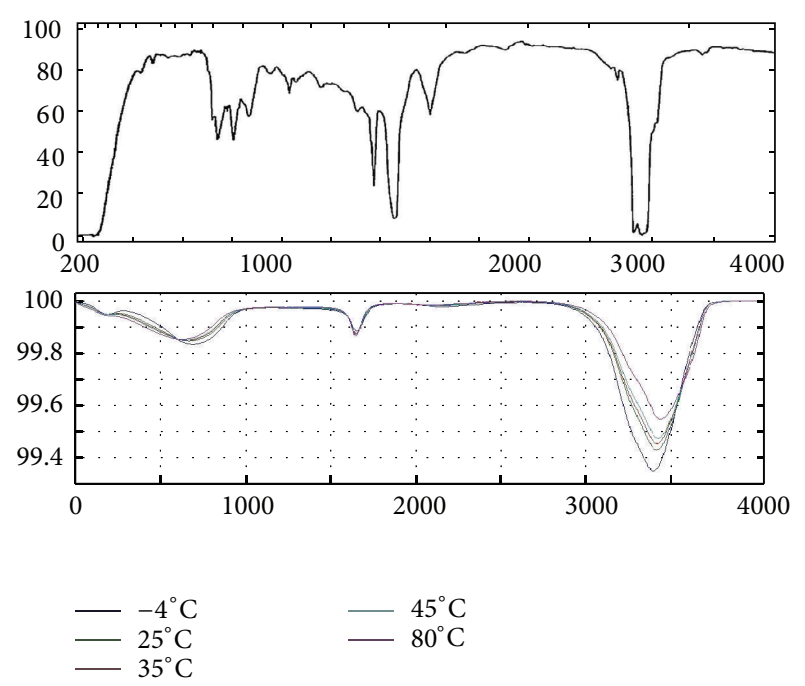

FIGURE 7: IR spectrum of heavy fuel oil as transmittance spectra [22] and the calculated absorption spectra of ordinary water (for 1micrometer thick samples).

oil; thus the analysis presented applies to most common types of crude oil.

It should be noted that the above-mentioned results for the absorption spectrums are derived by the Fourier transform infrared (FTIR) spectroscopy technique provided in [16].

Figure 7 shows a comparison between the behavior of crude oil and water in terms of transmittance in the 0 to 4000 wavenumber range which corresponds to the 0.1 to $120 \mathrm{THz}$ band we are investigating.

A transmittance-absorption conversion formula should be used to convert the results from an IR spectrum analysis to absorption spectra:

$$
A=2-\log _{10} T \text {. }
$$

Finally we can conclude that regarding the absorption loss in crude oil the frequency band from 70 to $85 \mathrm{THz}$ can be selected for transmission because it suffers min transmission loss and is also coincident with the ones observed for water.

3.4. Absorption Loss in Carbon Dioxide. Using the same approach as for water and crude oil, the absorption loss for $\mathrm{CO}_{2}$ is calculated as follows:

$$
L_{\mathrm{abs}}^{\mathrm{CO}_{2}}=10 \cdot k_{\mathrm{CO}_{2}} \cdot d \cdot \log _{10} e,
$$

where $k_{\mathrm{CO}_{2}}$ is defined using the essential FTIR for handling the Fourier transform of infrared data [16], and $d$ is the distance. Results for the $\mathrm{CO}_{2}$ absorption loss in $\mathrm{dB}$ as a function of distance and frequency in the transmission range from 70 to $85 \mathrm{THz}$ are given in Figure 8.

Investigating the $\mathrm{CO}_{2}$ absorption behavior is important because some compounds display higher absorption loss in some specific Terahertz bands. Our results prove that the $\mathrm{CO}_{2}$ loss can be neglected in the selected transmission window as it is less than $0.7 \mathrm{~dB}$ at $1 \mathrm{~m}$ (see Figure 8).

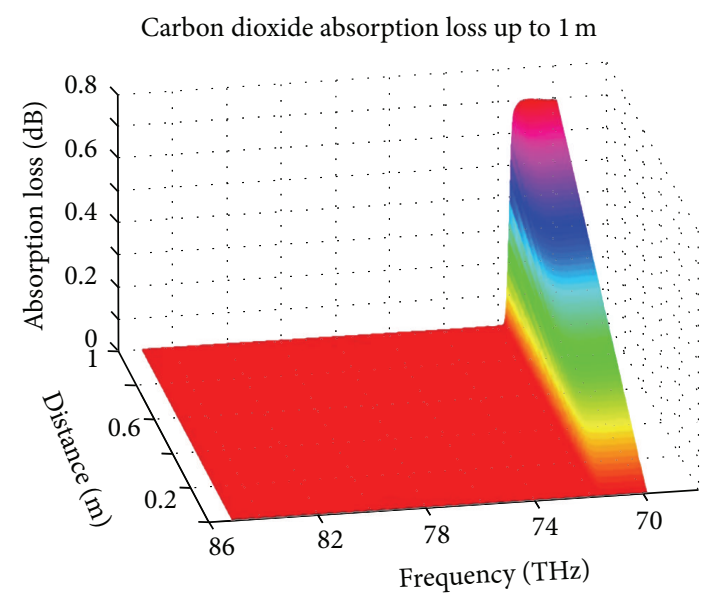

FIGURE 8: Infrared spectrum of $\mathrm{CO}_{2}$ absorption loss up to 1 meter in the selected transmission window.

3.5. Absorption Loss in Soil. For evaluating the soil path loss component, we can use the Peplinski principle, similar to the channel model for EM waves in soil medium described in [3]. However, that model has to be extended to cover the propagation of EM in soil in the Terahertz band. The absorption loss of the EM waves in soil medium $L_{\text {soil }}$ is given by (10), where $\alpha$ is the attenuation constant with the unit of $1 / \mathrm{m}$, and $\beta$ is the phase shifting constant with the unit of $\operatorname{radian} / \mathrm{m}$ :

$$
L_{\mathrm{abs}}^{\text {soil }}=20 \log _{10}(\beta)+8.69 \alpha d .
$$

The values of $\alpha$ and $\beta$ depend on the dielectric properties of soil:

$$
\begin{aligned}
& \alpha=2 \pi f \sqrt{\frac{\mu \epsilon^{\prime}}{2}\left[1+\left(\frac{\epsilon^{\prime \prime}}{\epsilon^{\prime}}\right)^{2}-1\right]}, \\
& \beta=2 \pi f \sqrt{\frac{\mu \epsilon^{\prime}}{2}\left[1+\left(\frac{\epsilon^{\prime \prime}}{\epsilon^{\prime}}\right)^{2}+1\right]},
\end{aligned}
$$

where $f$ is the operating frequency, $\mu$ is the magnetic permeability, and $\epsilon^{\prime}$ and $\epsilon^{\prime \prime}$ are the real and imaginary parts of the relative dielectric constant of the soil medium. They are calculated as follows:

$$
\begin{gathered}
\epsilon^{\prime}=1.15\left[1+\frac{\rho_{b}}{\rho_{s}}\left(\epsilon_{s}^{\alpha^{\prime}}\right)+m_{v}^{\beta^{\prime}} \epsilon_{\mathrm{fw}}^{\prime \alpha^{\prime}}-m_{v}\right]^{1 / \alpha^{\prime}}-0.68 \\
\epsilon^{\prime \prime}=\left[m_{v}^{\beta^{\prime \prime}} \epsilon_{\mathrm{fw}}^{\prime \prime \alpha^{\prime}}\right]^{1 / \alpha^{\prime}},
\end{gathered}
$$

where $m_{v}$ is the volumetric water content (VWC) of the soil medium, $\rho_{b}$ is the bulk density, $\rho_{s}=2.66 \mathrm{~g} / \mathrm{cm}^{3}$ is the specific density of the solid soil particles, $\alpha^{\prime}=0.65$ is an empirically determined constant, $\epsilon^{\prime}$ free water and $\epsilon^{\prime \prime}$ free water are the real and imaginary parts of the relative dielectric constant of water, and $\beta^{\prime}$ and $\beta^{\prime \prime}$ are empirically determined constants, dependent on soil composition in terms of sand and clay. 
In conclusion, based on the discussion in Sections 3.1, $3.2,3.3,3.4$, and 3.5 we can state that the frequencies from 76 to $80 \mathrm{THz}$ experience minimal absorption from all components that are common to the underground reservoirs. Thus, the above mentioned frequency band can be defined as a common transmission window suitable for realizing the communication between the wireless sensor nodes in the oil reservoir fractures. In the next section we present the model for estimating the propagation channel losses for this transmission window in the Terahertz range.

\section{Proposed Channel Model for EM Wave Propagation in Underground Oil Reservoirs}

Wireless transmission in a crude-oil-soil-water mixture using Terahertz EM waves exhibits quite different behavior as compared to traditional wireless communications and thus presents a multifaceted and challenging task. Models found in literature so far deal with the underground medium considering only the soil component, like for example the subsurface wireless channel model presented in [8] and the channel model for underground wireless sensor networks presented in [3].

The model we propose in this study allows the determination of the maximum transmission distance taking into account the total path loss and the operational frequency in the mixed environment of crude oil, water, $\mathrm{CO}_{2}$, and soil. Considering the complex composition of the crude oil and the high absorption of water, we have determined the existence of a viable transmission window in the Terahertz band that can be utilized for the communication process in this specific type of WUSNs.

The path loss of the Terahertz EM waves in oil reservoirs is due to five major factors and can be decomposed into five components: the path loss caused by signal spread $L_{\text {spread }}$, the absorption loss due to the water medium $L_{\text {water }}$, the absorption loss due to the crude oil $L_{\text {oil }}$, and absorption loss due to $\mathrm{CO}_{2}, L_{\mathrm{CO}_{2}}$, and finally the soil component, $L_{\text {soil }}$. Therefore,

$$
\begin{aligned}
& L_{\mathrm{EM}}= L_{\text {spread }}+L_{\mathrm{abs}}^{\mathrm{water}}+L_{\mathrm{abs}}^{\mathrm{oil}}+L_{\mathrm{abs}}^{\mathrm{CO}_{2}}+L_{\mathrm{abs}}^{\text {soil }}[\mathrm{dB}] \\
& \Downarrow \\
& L_{\mathrm{EM}}= 20 \cdot 10 \log _{10} \cdot 10\left(\frac{4 \pi \cdot f \cdot d}{c}\right) \\
&+k_{\text {water }}(f) \cdot d \cdot 10 \log _{10}(e) \\
&+k_{\mathrm{oil}}(f) \cdot d \cdot 10 \log _{10}(e) \\
&+k_{\mathrm{CO}_{2}}(f) \cdot d \cdot 10 \log _{10}(e) \\
&+6.4+20 \log (d)+20 \log (\beta)+8.69 \alpha d,
\end{aligned}
$$

where $f$ is the operating frequency; $d$ is the transmitting distance; and $c$ is the velocity of light in vacuum; $k_{\text {water }}(f)$ and $k_{\text {oil }}(f)$ are the absorption coefficients in water and crude oil, respectively. The value of $k_{\text {water }}(f)$ was discussed in Sections 3.1 and 3.2; the value of $k_{\text {oil }}(f)$ was discussed in Section 3.3

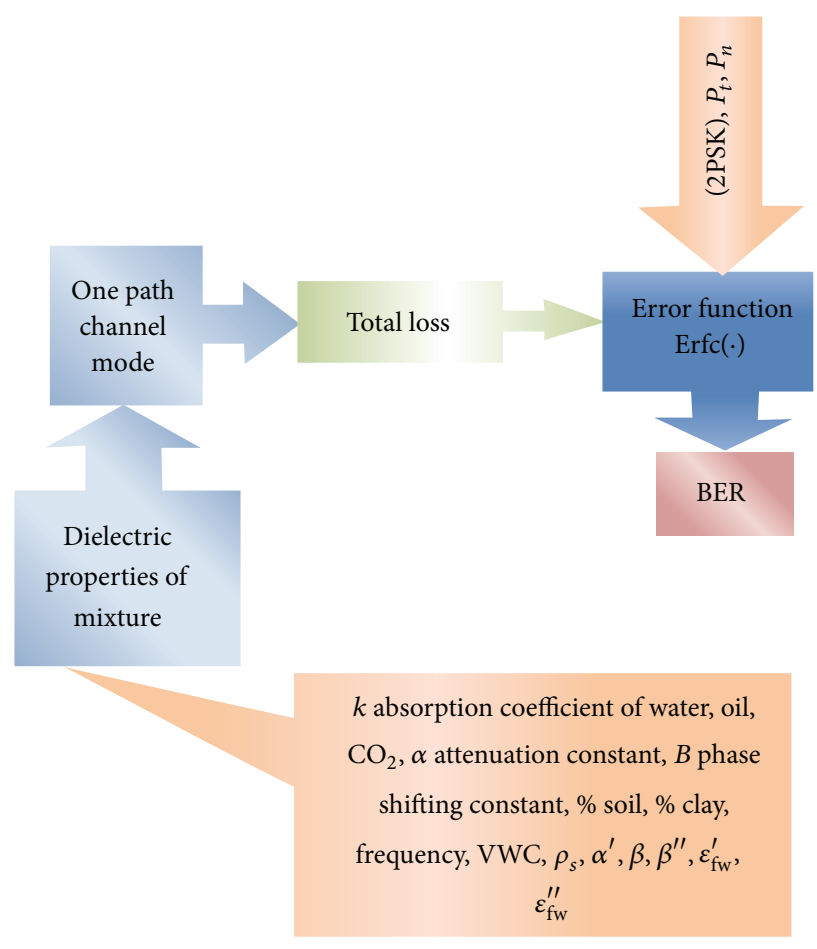

FIGURE 9: Propagation channel model for complex underground oil reservoirs.

and for most crude-oil types $k_{\text {oil }}(f)$ at room temperature is approximately 0.02 for the $70-85 \mathrm{THz}$ transmission window [17-24]; the value of $L_{\text {soil }}$ is determined according to (9); the value of $k_{\mathrm{CO}_{2}}$ is small enough to be neglected as discussed in Section 3.4. So, based on these, the absorption loss of the Terahertz EM waves in crude oil in the selected transmission window can be calculated using respective (1), (7), and (9). Finally, based on the above discussion and (13) a diagram of the suggested propagation channel model for complex underground oil reservoirs is presented in Figure 9.

Using the proposed channel model, we can numerically evaluate the total path loss of the Terahertz EM waves in the underground medium and provide estimation for the feasible transmission distances between the nodes as well as the incurred BER.

In the evaluations, we utilize the assumptions made in Section 3. Regarding the effect of the soil component, the parameters are chosen to reflect typical conditions as reported in [3]. VWC is set to $5 \%$, the sand particle percent to $50 \%$, the clay percent to $15 \%$, and the bulk density to $1.5 \mathrm{~g} / \mathrm{cm}^{3}$. The operating frequency is chosen between 70 and $85 \mathrm{THz}[1]$. For the investigation of the channel through a mixed composition of soil and water/oil the SNR is given by SNR $=P_{t}-L_{f}-P_{n}$, where $P_{t}$ is the transmit power, $L_{f}$ is the total path loss, and $P_{n}$ is the noise energy. For our evaluations $P_{t}$ is varied between 10 and $30 \mathrm{dBm}$ and $P_{n}$ is $-103 \mathrm{dBm}$ [3]. Similarly, even though the noise, $P_{n}$, may change depending on the properties of the soil mixture, the value taken is a representative value which can be used for the calculations of the BER. 


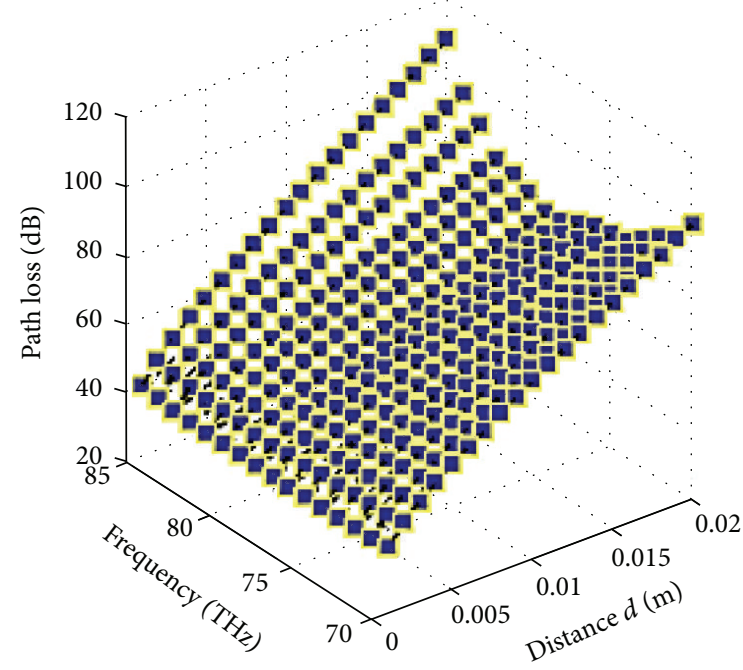

(a)

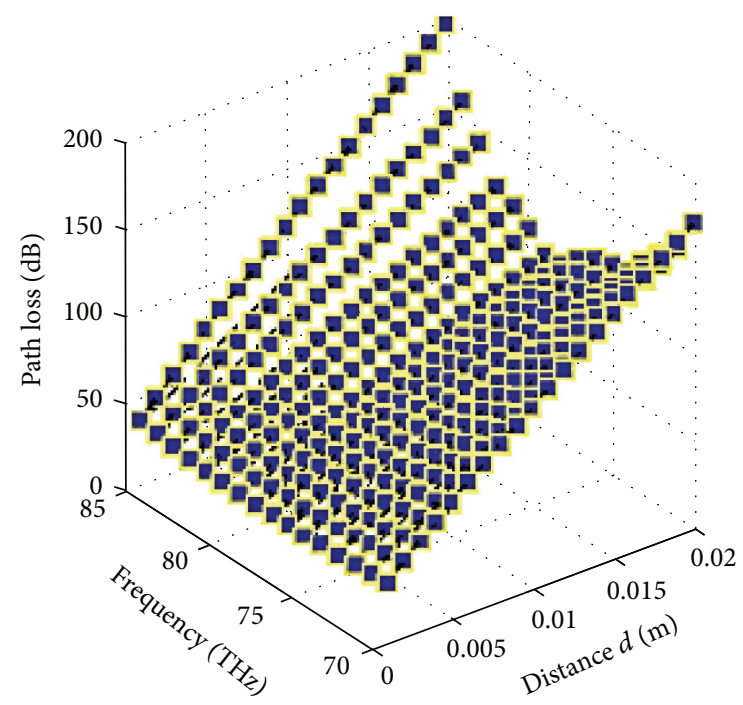

(c)

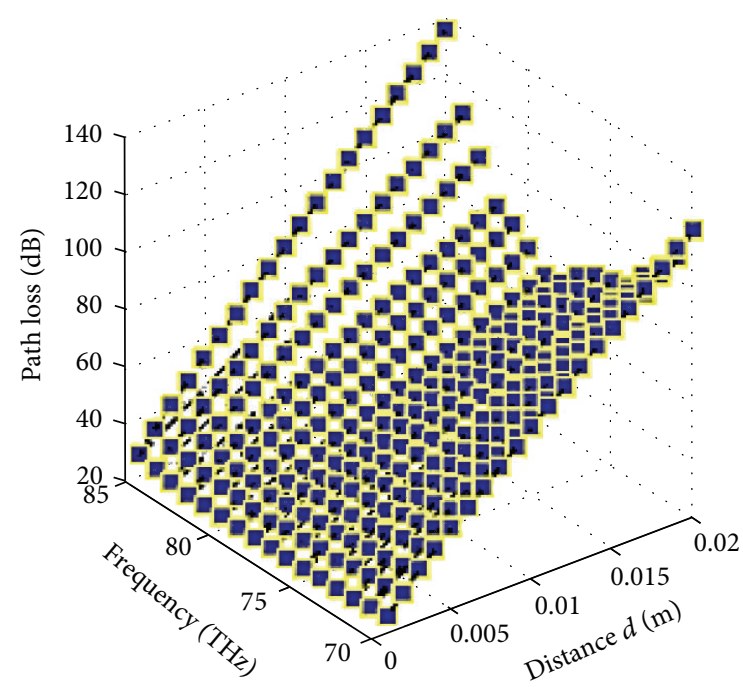

(b)

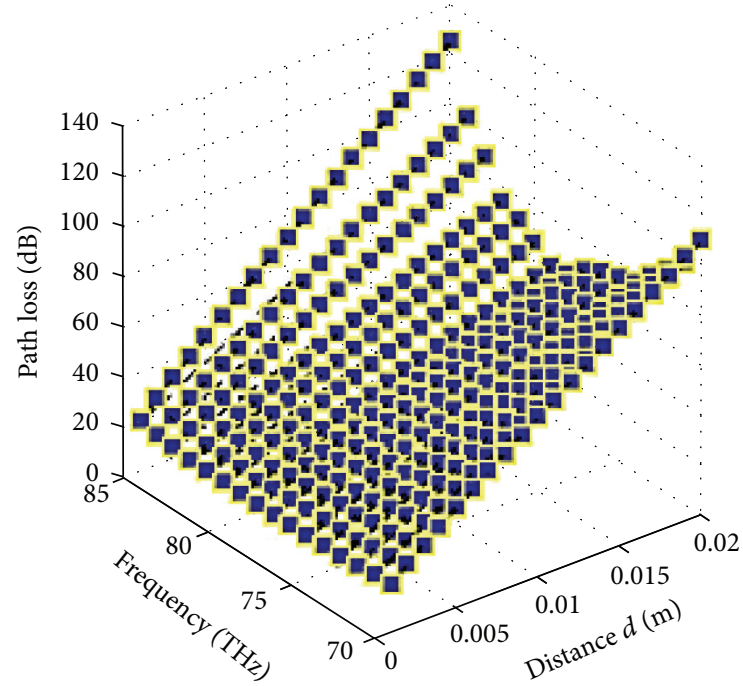

(d)

Figure 10: Path loss versus operating frequency and transmission distance in mixtures (a), (b), (c), and (d).

The BER also depends on the modulation method. In order to provide an initial investigation in this area, various modulation methods were studied and the PSK modulation was chosen since it has been proven in $[1-3]$ that the PSK modulation method provides the longest communication range. For 2PSK, the BER as a function of the SNR is given as $\mathrm{BER}=0.5 \operatorname{erfc}(\sqrt{\operatorname{SNR}})$, where $\operatorname{erfc}(\cdot)$ is the error function and SNR is the signal to noise ratio.

Four mixtures with different mixture ratios were investigated as suggested by the authors in [25-27]. Specifically these are
(a) $\% 10$ water/oil $+\% 50 \mathrm{CO}_{2}+\% 40$ soil,
(b) $\% 20$ water/oil $+\% 70 \mathrm{CO}_{2}+\% 10$ soil,
(c) $\% 30$ water $/$ oil $+\% 60 \mathrm{CO}_{2}+\% 10$ soil,

\section{(d) $\% 20$ water $/$ oil $+\% 80$ soil.}

The results in Figure 10 give the estimation of the expected path loss in the range of 70 to $85 \mathrm{THz}$ for these mixtures. It can be seen that "mixture (c)" has the highest path loss, while "mixtures (a), (b), and (d)" exhibit much lower path loss values, which is due to the much lower content of water.

Figure 11 provides the estimation of the incurred BER in the operating range from 70 to $85 \mathrm{THz}$ for distances up to $10 \mathrm{~cm}$, where the transmission power is set to $10 \mathrm{~dB}$.

The results for transmission power $30 \mathrm{~dB}$ are presented in Figure 12. Even though the increased transmission power provides a slightly longer communication range between the nodes, the most important conclusion we can make is that there exists a very close relationship between the transmission 


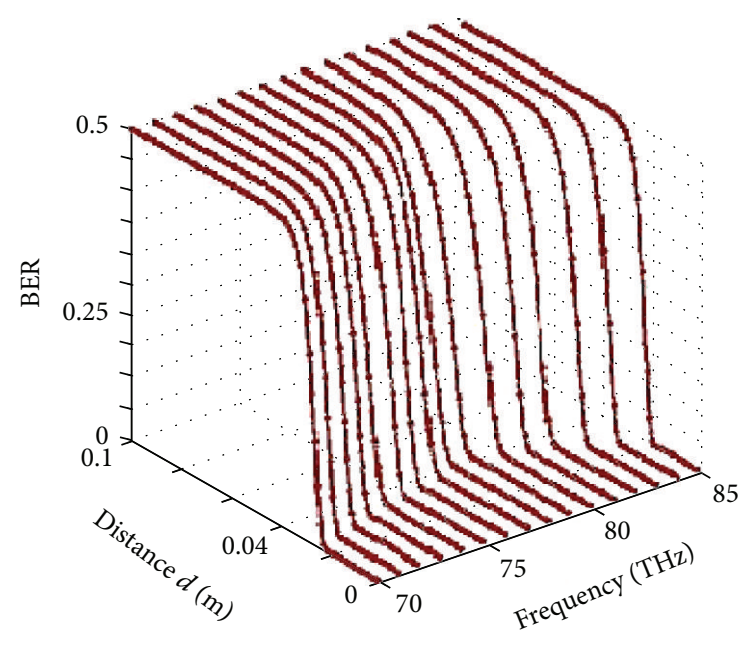

(a)

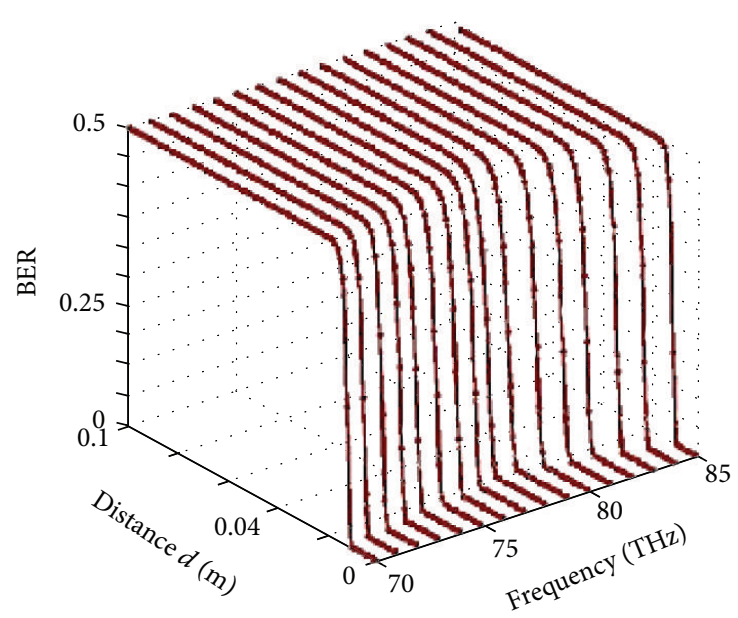

(c)

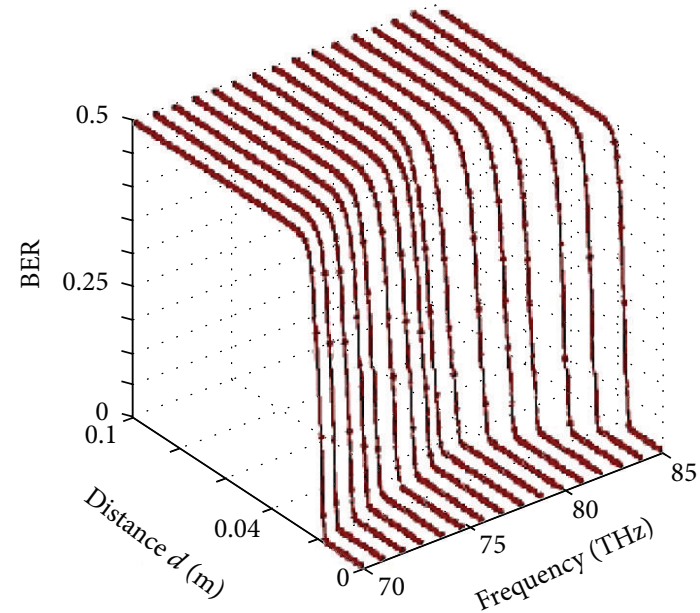

(b)

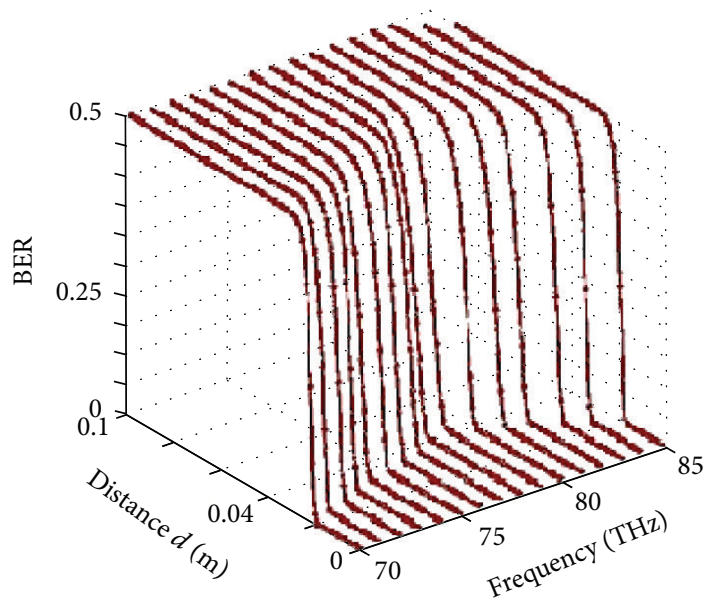

(d)

FIGURE 11: BER versus operating frequency and transmission distance for mixtures (a), (b), (c), and (d) with $10 \mathrm{dBm}$ transmit power.

distance, the frequency, and the soil composition. Major problem is the high water content in the soil mixture (compare "mixture (c)" with "mixtures (a), (b), and (d)").

\section{Channel Capacity for Terahertz WUSNs}

Finally the channel capacity available between two underground sensor nodes in the EM-based WUSNs can be theoretically evaluated. We consider the classic channel capacity formula given by

$$
C=B \cdot \log _{10}\left(1+\frac{P_{t} \cdot L_{p}}{N}\right),
$$

where $C$ is the channel capacity; $B$ is the bandwidth of the channel; $P_{t}$ is the transmission power; $L_{p}$ is the path loss; and $N$ is the total noise and interference power. In traditional EM wave-based wireless channel, the bandwidth $B$ is a constant and previously determined by the specific applications, while the channel path loss $L_{p}$ is an exponential function of the transmission distance. In our case the $L_{p}$ is calculated according to (13); transmission power is assumed to be $10 \mathrm{dBm}$ as supported by sensor datasheets given in [28].

Figure 13 shows the details for the channel capacity, in Kbits/s zoomed at specific frequencies 78, 79, and $80 \mathrm{THz}$. Considering both transmission distance and channel capacity it can be pointed out that "mixture (a)" outperforms the other mixtures. Also it is important to note that the optimal results for all soil-crude-oil-water mixtures considered typical for the oil reservoir are at transmission frequency $79 \mathrm{THz}$.

Based on the investigation and the numerical results presented so far we can state that, for WUSNs, operating in a mixture of soil, water, crude oil, and $\mathrm{CO}_{2}$, EM waves in the Terahertz band can be used to provide communication for a distance of up to $7 \mathrm{~cm}$. This type of sensor networks can be used not only for oil recovery but also for detection of forthcoming earthquakes as well as extraction of other underground resources. Generally these are large scale projects, where the value of the final product, the information provided, and the length of operation justify the high expenses incurred by the sensors themselves and their deployment. 


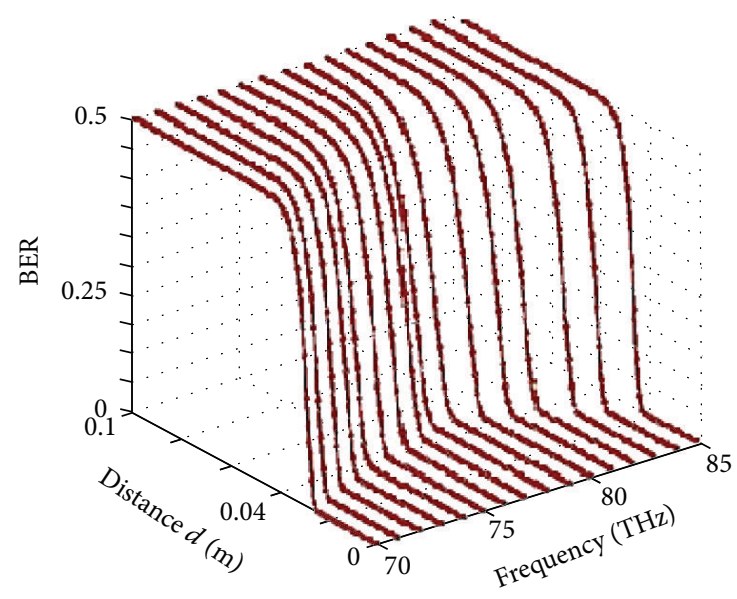

(a)

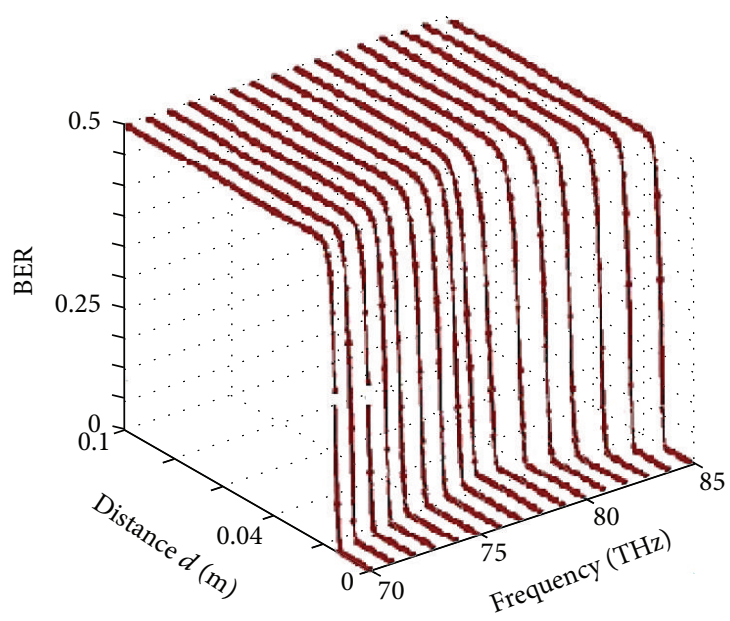

(c)

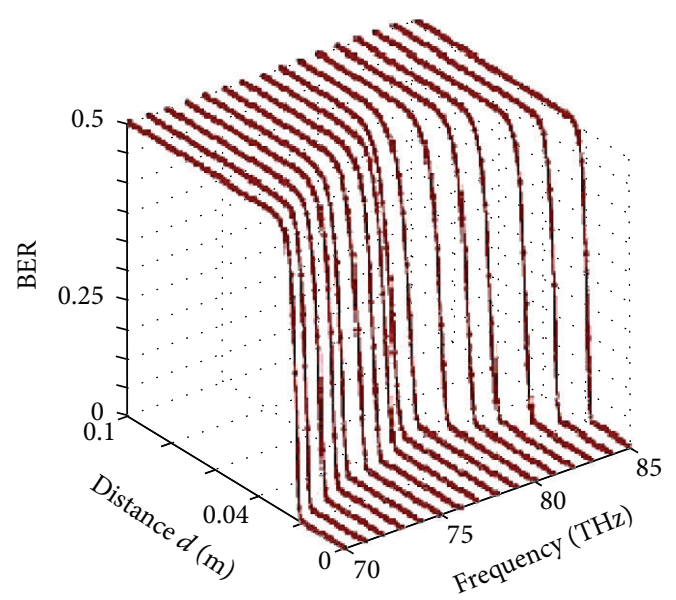

(b)

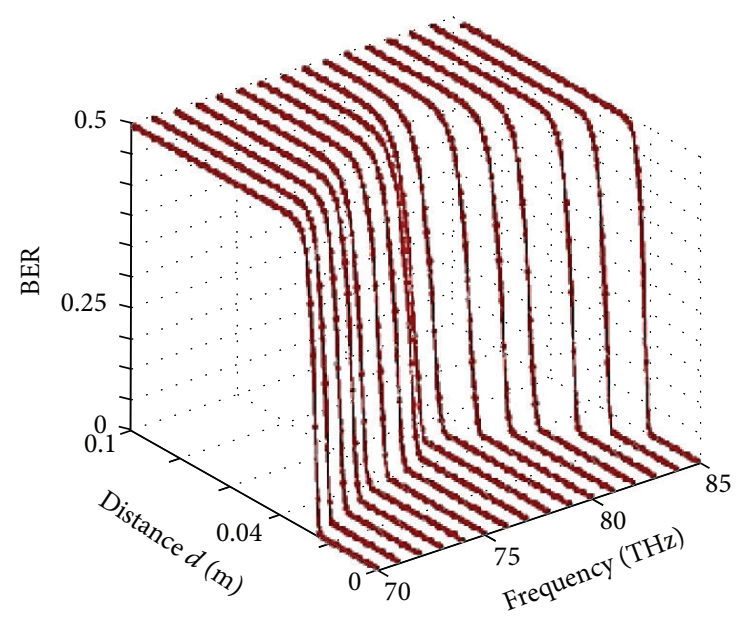

(d)

FIGURE 12: BER versus operating frequency and transmission distance for mixtures (a), (b), (c), and (d) with for $30 \mathrm{dBm}$ transmit power.

\section{Conclusion}

In this paper, we have addressed the subject of EM-based WUSNs communication techniques and studied the question whether they can enable efficient communication in complex underground environments. Accordingly, we have determined a suitable transmission range in the $\mathrm{THz}$ band which is optimal in terms of the absorption losses of the different ingredients comprising the complex underground oil reservoir environment. Furthermore a comprehensive channel model is proposed for EM-based WUSNs operating in mixed crude oil/soil/water and $\mathrm{CO}_{2}$ medium. Analyzing in detail the propagation properties of EM waves in the underground environment, a novel communication channel model, based on the EM channel characteristics is developed and its performance in terms of BER for different soil mixtures is investigated. The first mixture considered is that of $\% 10$ water/oil $+\% 50 \mathrm{CO}_{2}+\% 40$ soil, the second is that of $\% 20$ water/oil $+\% 70 \mathrm{CO}_{2}+\% 10$ soil, the third mixture is $\% 30$ water/oil $+\% 60 \mathrm{CO}_{2}+\% 10$ soil, and the forth one is 20 water/oil $+\% 80$ soil. Our results show that the Terahertz communication channel has a strong dependence on both the molecular composition of the medium and the transmission distance. The main factor affecting the behavior of the EM waves in the Terahertz band is the absorption caused by water. In the short range, that is, for a transmission distance in the order of up to $7 \mathrm{~cm}$, the Terahertz band can be considered as a single transmission window almost $15 \mathrm{THz}$ wide. Even a narrow window from 76 to $80 \mathrm{THz}$ band still provides high enough channel capacity to support sufficiently high transmission rates. The very high channel capacity of the Terahertz band not only does support very high transmission bit rates, but also enables new information encoding and modulation techniques as well as novel cross layer networking protocols which will allow tuning of the performance based on different physical layer parameters. In this respect, our work, which evaluates the specifics of the underground propagation channel, presents a guideline for other researches that will be working in the promising new field of WUSNs. 


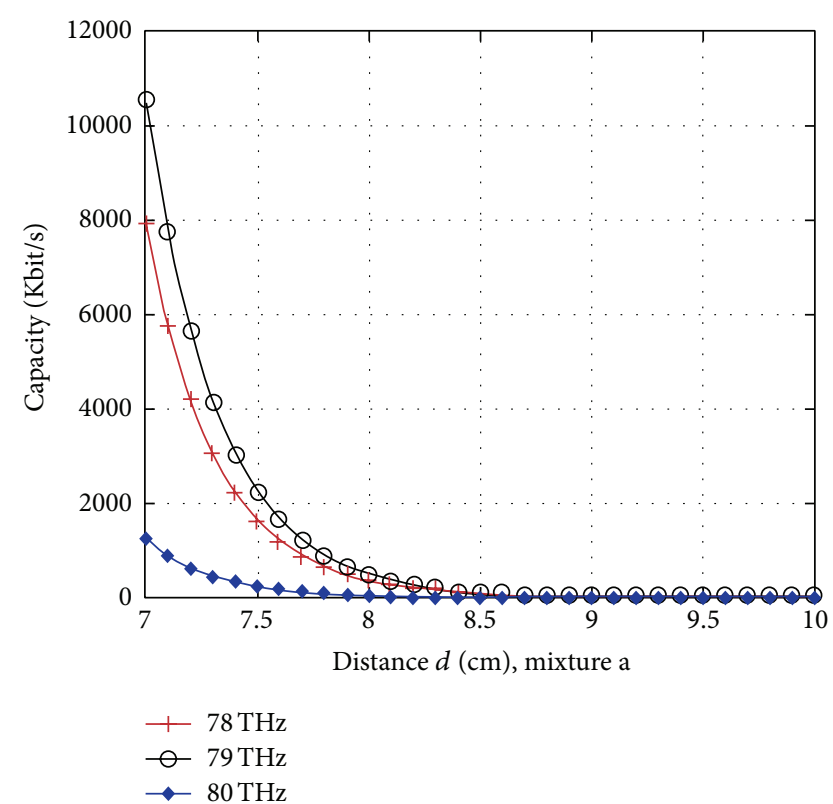

(a)

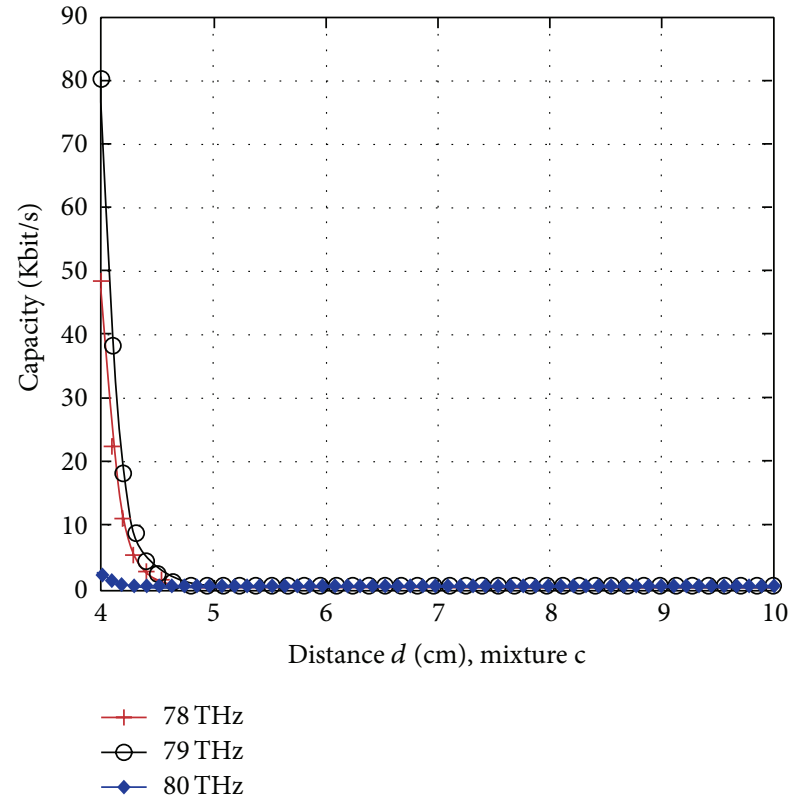

(c)

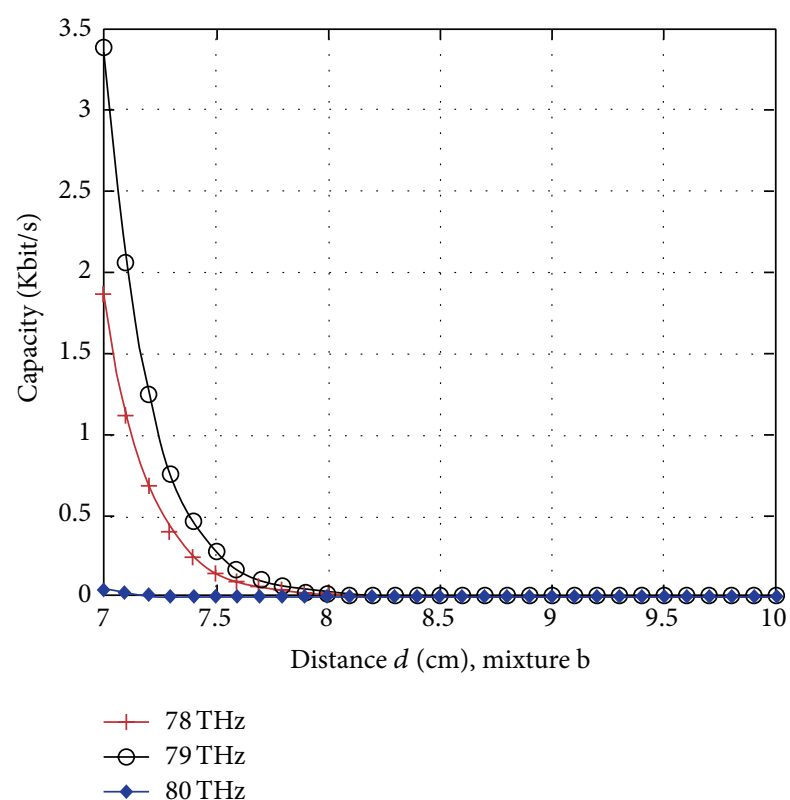

(b)

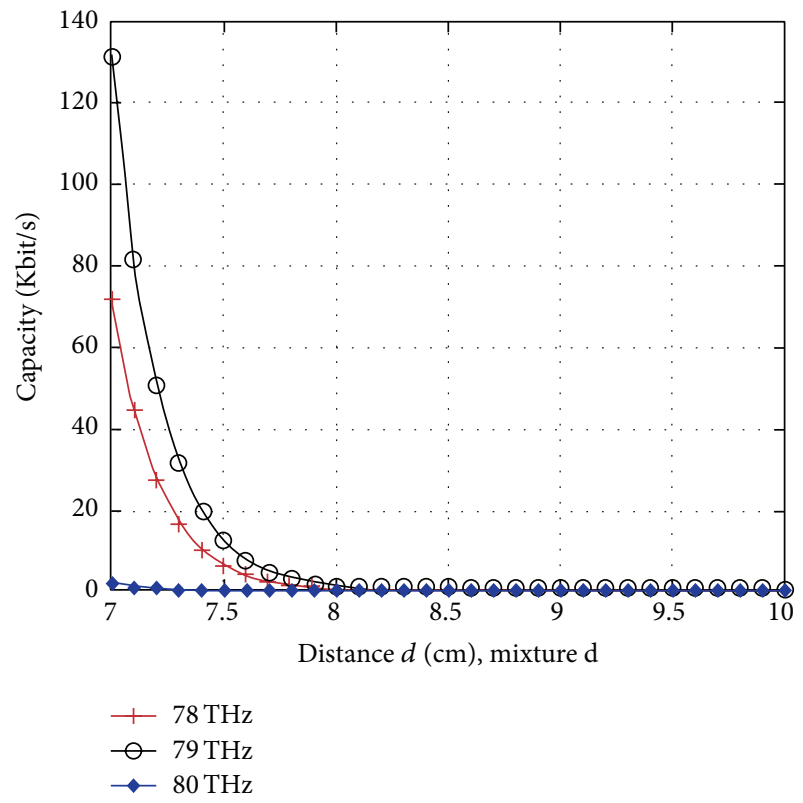

(d)

FIGURE 13: Capacity of the channel versus distance at $78 \mathrm{THz}, 79 \mathrm{THz}$, and $80 \mathrm{THz}$ for mixtures (a), (b), (c), and (d) with $10 \mathrm{dBm}$ transmit power.

\section{Conflict of Interests}

The authors declare that there is no conflict of interests regarding the publication of this paper.

\section{References}

[1] M. A. Akkaş, I. F. Akyildiz, and R. Sokullu, “Terahertz channel modeling of underground sensor networks in oil reservoirs," in Proceedings of the IEEE Global Communications Conference (GLOBECOM '12), pp. 543-548, Anaheim, Calif, USA, December 2012.

[2] I. F. Akyildiz, Z. Sun, and M. C. Vuran, "Signal propagation techniques for wireless underground communication networks," Physical Communication, vol. 2, no. 3, pp. 167-183, 2009.

[3] M. C. Vuran and I. F. Akyildiz, "Channel model and analysis for wireless underground sensor networks in soil medium," Physical Communication, vol. 3, no. 4, pp. 245-254, 2010. 
[4] N. Liu, R. Paranjape, and K. Asghari, "Underground ultrasound probing for monitoring carbon dioxide flooding in oil producing reservoirs," in Proceedings of the IEEE Canadian Conference on Electrical \& Computer Engineering, Winnipeg, Canada, May 2002.

[5] X. Xu, Y. Yongqiang, Y. Cao, and L. Wei, "The research and design of oil field associated gas recovery device," in Proceedings of the International Conference on Electronic and Mechanical Engineering and Information Technology (EMEIT '11), pp. 10061008, August 2011.

[6] F. Mazzini, D. Kettler, J. Guerrero, and S. Dubowsky, “Tactile robotic mapping of unknown surfaces, with application to oil wells," IEEE Transactions on Instrumentation and Measurement, vol. 60, no. 2, pp. 420-429, 2011.

[7] A. R. Silva, Channel characterization for wireless underground sensor networks [M.S. thesis], University of Nebraska, Lincoln, Neb, USA, 2010.

[8] Z. Sun and B. Zhu, "Channel and energy analysis on magnetic induction-based wireless sensor networks in oil reservoirs," in Proceedings of the IEEE International Conference on Communications (ICC '13), pp. 1748-1752, Budapest, Hungary, June 2013.

[9] M. Peng, Y. Li, T. Q. S. Quek, and C. Wang, "Device-Deviceto-device underlaid cellular networks under rician fading channels," IEEE Transactions on Wireless Communications, vol. 13, no. 8, pp. 4247-4259, 2014.

[10] M. Kyrö, K. Haneda, J. Simola, K.-I. Takizawa, H. Hagiwara, and P. Vainikainen, "Statistical channel models for $60 \mathrm{GHz}$ radio propagation in hospital environments," IEEE Transactions on Antennas and Propagation, vol. 60, no. 3, pp. 1569-1577, 2012.

[11] Y. Maréchal, “The molecular structure of liquid water delivered by absorption spectroscopy in the whole IR region completed with thermodynamics data," Journal of Molecular Structure, vol. 1004, no. 1-3, pp. 146-155, 2011.

[12] J.-J. Max and C. Chapados, "Isotope effects in liquid water by infrared spectroscopy. III. $\mathrm{H}_{2} \mathrm{O}$ and $\mathrm{D}_{2} \mathrm{O}$ spectra from 6000 to $0 \mathrm{~cm}^{-1}$," Journal of Chemical Physics, vol. 131, no. 18, Article ID 184505, 2009.

[13] $\varnothing$. A. Tengesdal, Measurement of seawater refractive index and salinity by means of optical refraction [M.S. thesis], University of Bergen, 2012.

[14] S. Yamauchi, K. Takayama, Y. Imai, K. Takeya, and M. Tonouchi, "Dielectric behavior of water in $\mathrm{THz}$ influenced by alkali and alkaline-earth halides," in Proceedings of the 35th International Conference on Infrared, Millimeter, and Terahertz Waves (IRMMW-THz '10), Rome, Italy, September 2010.

[15] M. A. Akkas and R. Sokullu, "A channel model for underground sensor networks in the mixture of crude oil, water and soil," Journal of Communication and Computer, vol. 10, no. 10, pp. 1333-1349, 2013.

[16] http://www.essentialftir.com.

[17] S. L. Silva, A. M. S. Silva, J. C. Ribeiro, F. G. Martins, F. A. Da Silva, and C. M. Silva, "Chromatographic and spectroscopic analysis of heavy crude oil mixtures with emphasis in nuclear magnetic resonance spectroscopy: a review," Analytica Chimica Acta, vol. 707, no. 1-2, pp. 18-37, 2011.

[18] G. Pérez-Caballero, J. M. Andrade, S. Muniategui, and D. Prada, "Comparison of single-reflection near-infrared and attenuated total reflection mid-infrared spectroscopies to identify and monitor hydrocarbons spilled in the marine environment," Analytical and Bioanalytical Chemistry, vol. 395, no. 7, pp. 23352347, 2009.
[19] P. de Peinder, D. D. Petrauskas, F. Singelenberg et al., "Prediction of long and short residue properties of crude oils from their infrared and near-infrared spectra," Applied Spectroscopy, vol. 62, no. 4, pp. 414-422, 2008.

[20] J. S. Basta, "Spectroscopic studies on some crude oils from the North Western Desert, Egypt," Petroleum Science and Technology, vol. 28, no. 8, pp. 779-789, 2010.

[21] L. Shaoping, D. Luoyong, and D. Qingxin, "Study on the processes for lubricant base oil from oman and cabinda blended crude oil no. 3 fraction," Petroleum Science and Technology, vol. 28, no. 11, pp. 1129-1139, 2010.

[22] P. de Peinder, T. Visser, D. D. Petrauskas, F. Salvatori, F. Soulimani, and B. M. Weckhuysen, "Partial least squares modeling of combined infrared, ${ }^{1} \mathrm{H}$ NMR and ${ }^{13} \mathrm{C}$ NMR spectra to predict long residue properties of crude oils," Vibrational Spectroscopy, vol. 51, no. 2, pp. 205-212, 2009.

[23] P. I. Rosales, M. T. Suidan, and A. D. Venosa, "A laboratory screening study on the use of solidifiers as a response tool to remove crude oil slicks on seawater," Chemosphere, vol. 80, no. 4, pp. 389-395, 2010.

[24] R.-M. Bao, L. Tian, K. Zhao, S.-M. Zhu, L.-M. Liu, and G.Z. Zhao, "Spectroscopy studies on the Tuha crude oil in the Terahertz range," in International Symposium on Photoelectronic Detection and Imaging 2011: Terahertz Wave Technologies and Applications, vol. 8195 of Proceedings of SPIE, Beijing, China, May 2011.

[25] E. Tzimas, C. Garcia-Cortes, and S. D. Peteves, "Enhanced oil recovery using carbon dioxide in the European energy system," European Commission Technical Report 21895, DirectorateGeneral for Joint Research Centre, 2005.

[26] F. Gozalpour, S. R. Ren, and B. Tohidi, " $\mathrm{CO}_{2}$ EOR and storage in oil reservoirs," Oil and Gas Science and Technology, vol. 60, no. 3, pp. 537-546, 2005.

[27] W. Schulte, "Experience for use in $\mathrm{CO}_{2}$ for enhanced oil recovery in the USA," in Proceedings of the CO2 Conference, 2004.

[28] http://www.microstrain.com/wireless. 

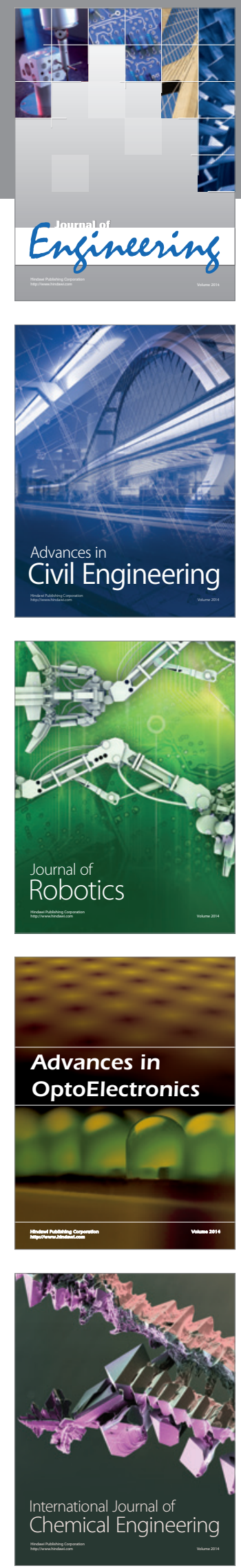

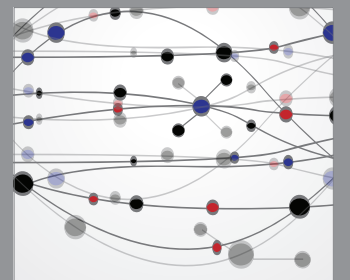

The Scientific World Journal
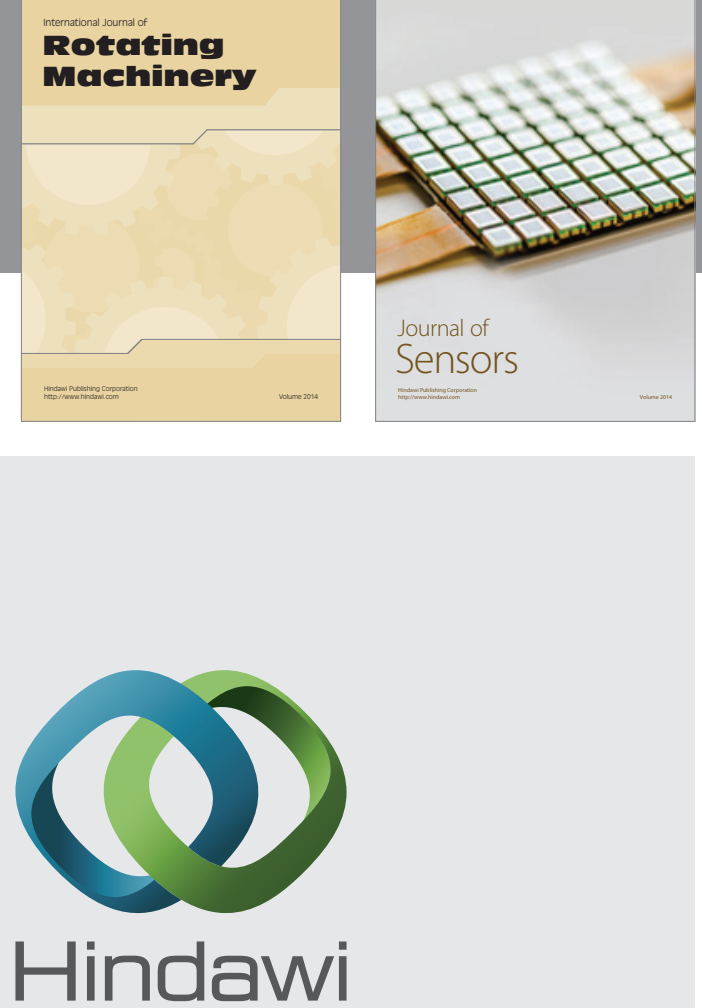

Submit your manuscripts at http://www.hindawi.com
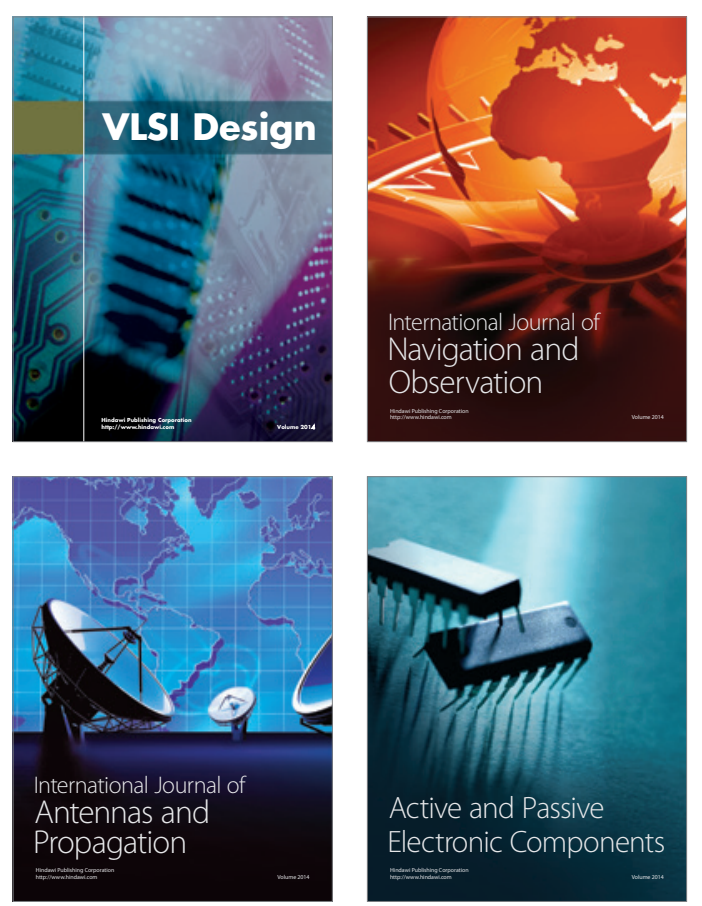
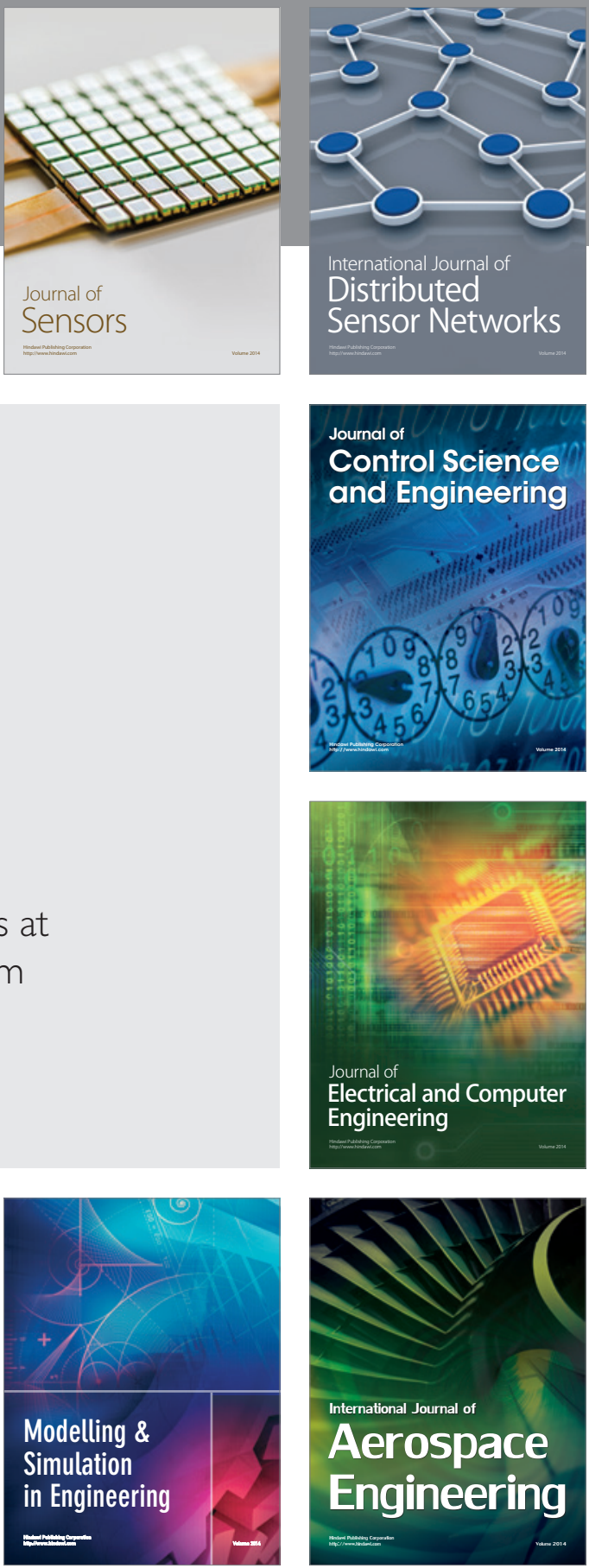

Journal of

Control Science

and Engineering
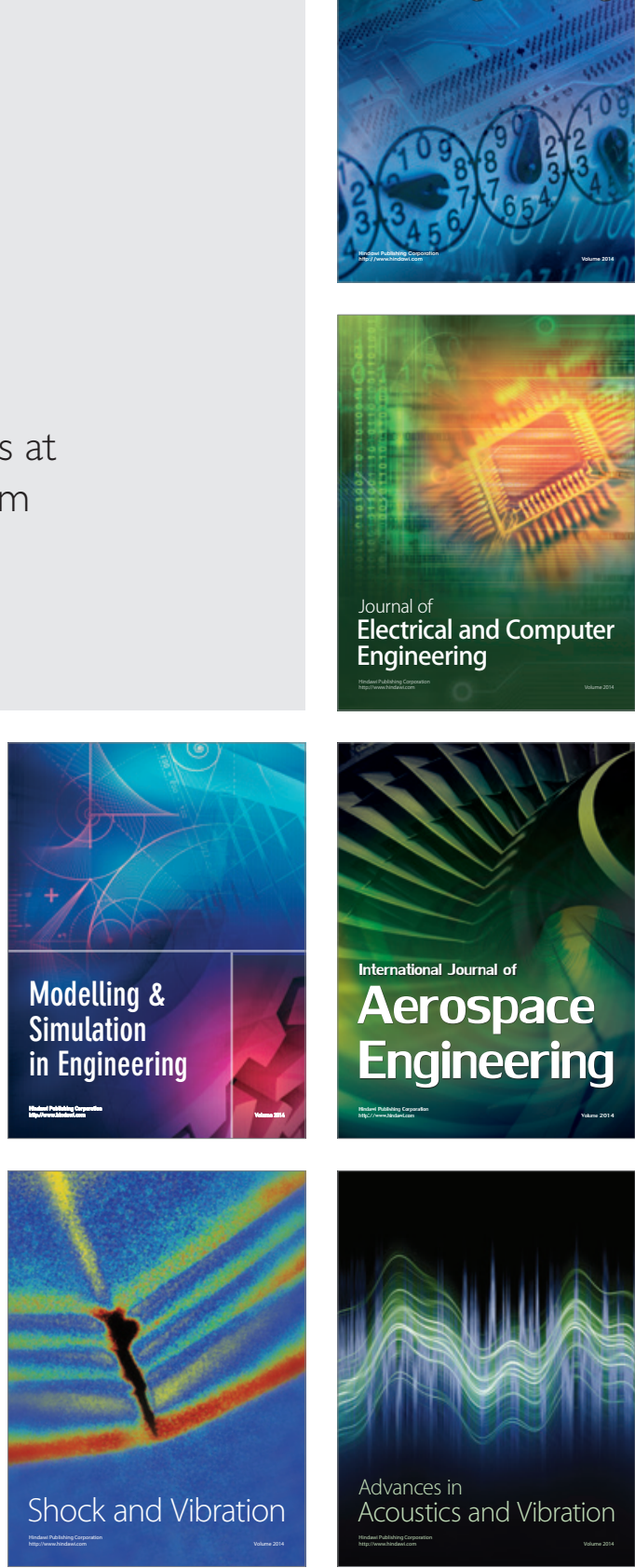